\title{
AN ENGINEERING OVERVIEW OF AN ELECTROSTATIC QUADRUPOLE LATTICE FOR A HIGH CURRENT TRANSPORT EXPERIMENT*
}

\author{
Victor Karpenko ${ }^{\dagger}$, Peter Seidl', Lawrence Berkeley National Laboratory, 1 Cyclotron Rd, Berkeley, CA 94720, USA \\ R. Mark Franks ${ }^{\dagger}$, Steven Lund ${ }^{\dagger}$ Lawrence Livermore National Laboratory, PO Box 808, Livermore, CA 95531, USA
}

\begin{abstract}
The Heavy Ion Fusion Virtual National Laboratory is building a High Current Experiment (HCX) to explore the transport of a heavy-ion beam at a scale appropriate to the low-energy end of a driver for heavy ion fusion. The HCX is presently being designed and assembled in multiple stages at the Lawrence Berkeley National Laboratory (LBNL). The scientific motivation for the HCX is presented in a related paper [1]. Here, we overview the engineering design of an early phase of the HCX involving the transport of a $1.7 \mathrm{MeV}, 0.7 \mathrm{Amp} \mathrm{K}^{+}$beam through a lattice of alternating gradient electrostatic quadrupoles (ESQs). Four tanks of 10 quadrupoles each are ultimately planned, corresponding to 20 periods of ESQ transport. Mechanical, high-voltage, and vacuum engineering aspects of the ESQ transport lattice are presented.
\end{abstract}

\section{INTRODUCTION}

The objective of the HCX is to explore beam transport limitations in a regime of line-charge density $(\lambda \sim 0.2$ $\mu \mathrm{C} / \mathrm{m})$ and pulse duration $(\tau \sim 3-10 \mu \mathrm{s})$ relevant to that typically envisioned for full-scale drivers. A single-beam rather than a multi-beam transport channel is employed to economically address the intrinsic transport limits associated with space charge dominated ion-beams. The HCX will explore intense ion-beam transport limits and loss in beam quality (emittance growth) resulting from applied field nonlinearities; misalignment-induced centroid errors and steering; imperfect vacuum and background gas; halo and electron effects resulting from lost particles; etc. The HCX will provide new data on acceptable beam filling factors in the machine aperture under realistic, rather than scaled, conditions expected in the low-energy end of a driver. This data will be valuable as the filling factor strongly influences the transverse dimensions of multi-beam transport channels that will ultimately be employed in future research facilities and a driver. The transverse dimension in-turn plays a strong role in the volume, therefore expense, of cores for induction acceleration and compression of the multiplebeams.

Early phases of this project (Phase I) detailed here involves the transport of a coasting beam (no acceleration or compression) through a lattice of ESQs arranged in a

\footnotetext{
* This work supported by the Office of Energy Research, U.S. Department of Energy, at Lawrence Berkeley National Laboratory and Lawrence Livermore National Laboratory under contract numbers DEAC03-76SF00098 and W-7405-Eng-48.

$\dagger$ Heavy Ion Fusion Virtual National Laboratory

mail to: paseidl@lbl.gov
}

symmetric FODO channel. Each of the four tanks making up the transport lattice contains ten ESQs and has provisions for beam matching and steering, diagnostics, and variable quadrupole skew coupling via one rotatable quadrupole. An alignment and support scheme is employed that positions the quadrupole strings independently of the high vacuum vessel $\left(\sim 10^{-7}\right.$ Torr $)$. The HCX employs an ESQ injector and a six ESQ matching section that have been retrofit for this application. The injector is detailed in another paper [2]. Here we focus on the units making up the ESQ transport lattice of the Phase I HCX.

\section{ELECTROSTATIC QUADRUPOLES}

\subsection{Mechanical Design Aspects}

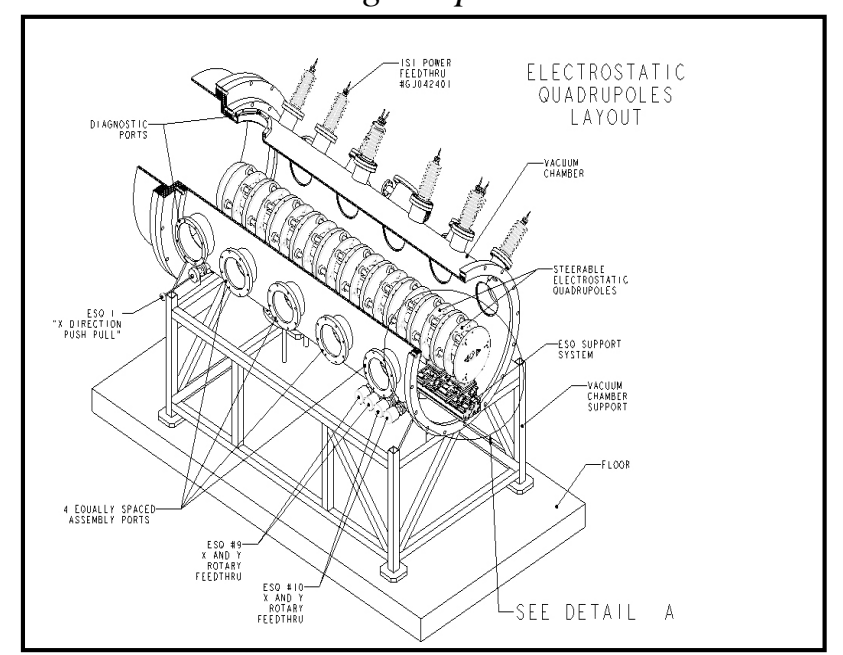

Figure 1: The vacuum vessel is shown mounted to a conceptual support strǘctưfre! 'ESQ's are mounted on a common rail to facilitate alignment.

The HCX ion beam will be transported through a periodic FODO lattice of precision aligned ESQs with lattice half-periods of $\mathrm{L}=217.6 \mathrm{~mm}$ and a clear bore radius of $r_{p}=23 \mathrm{~mm}$ (Fig. 2). Each ESQ consists of four cylindrical electrodes of radius (8/7) $\mathrm{r}_{\mathrm{p}}$ and length 154.51 $\mathrm{mm}$ with radiused ends. The electrodes are sized to eliminate lowest-order multipole field errors in 2D crosssection. Two electrodes (oriented horizontally) are biased to potential $+\mathrm{V}$ and are supported by one end-plate with the opposing two electrodes biased to $-\mathrm{V}$ (oriented vertically) supported by the opposing end-plate. The 12.7 $\mathrm{mm}$ thick end-plates, with their respective electrodes, are supported and aligned by precision ground alumina insulating rods attached to a ground ring at the center of the ESQ. Features are placed into the assembly by $\mathrm{CNC}$ 
$\pm 75 \mu \mathrm{m}$ relative to the centerline of the beam to meet a $\pm 100 \mu \mathrm{m}$ tolerance goal.

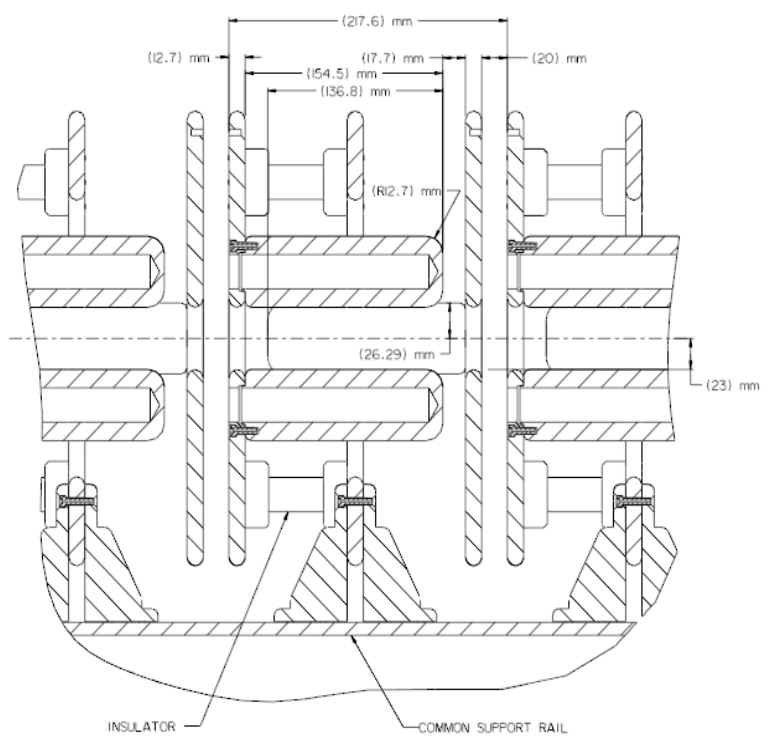

Figure 2: A cross-section view of the ESQ lattice showing critical dimensions and the common rail support.

\subsection{High Voltage Design Aspects}

Individual ESQ electrodes will be biased in a bipolar configuration to $\mathrm{V} \sim \pm 60 \mathrm{kV}$ using commercially available feedthrough connectors and dc power supplies. Biases of adjacent ESQs will follow an alternating gradient pattern. The voltages applied to the last 4 ESQs mounted on the rail will be independently adjustable to allow re-matching of the beam envelope at the end of each tank. The first quadrupole is also independently biased and the $2^{\text {nd }}-6^{\text {th }}$ ESQs will be connected in parallel with alternating gradient symmetry to reduce the number of feedthrough connectors. All metal components of the ESQ are machined from 304 stainless steel to a $32 \mu$ inch finish followed by electropolishing to maintain peak surface fields of less than $100 \mathrm{kV} / \mathrm{cm}$. Edges in the ESQ have been radiused to reduce edge-field enhancements. The alumina struts are protected from breakdown by shielding the triple point from high electric fields. A prototype ESQ assembly was successfully tested to $\pm 120 \mathrm{kV}$ without breakdown.

\subsection{Assembly and Pre-Alignment}

The ESQs are assembled and pre-aligned on a common rail (Fig. 3) relative to an established centerline using conventional mechanical gauges and optical techniques common to accelerators. This alignment is done prior to installation in the vacuum vessel with a final check following installation.

The rail is supported at its Airy point using a classic three point kinematic system to minimize deviations from straightness and provide the required degrees of freedom. Each of the three support points have two contact points steel flats that are bonded to the stainless steel mounting block. Two non-contacting spring-loaded retaining pins provide seismic restraint to the rail at each support point.

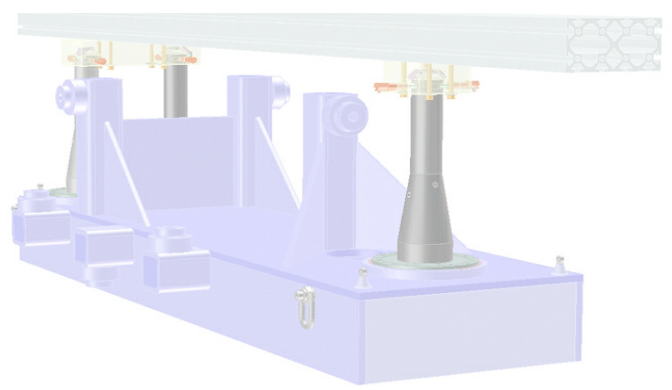

Figure 3: Common rail mounted on the kinematic system with spring-loaded pins to provide seismic restraint.

\section{ALIGNMENT SYSTEM}

The ESQ rail is attached to an external support structure through three welded bellows feedthroughs, decoupling it from the vacuum vessel and other loads. The alignment system utilizes a "six-strut system" [3] of six orthogonal links (struts) to provide six degrees of adjustment freedom for external alignment (Fig. 4). Verification features are also incorporated. These struts provide kinematic support to allow easy and precise alignment of the ESQ rail, minimize vibrations, and meet seismic requirements. The struts have ball jointed end connections that are arranged orthogonally to simplify position adjustment. The system

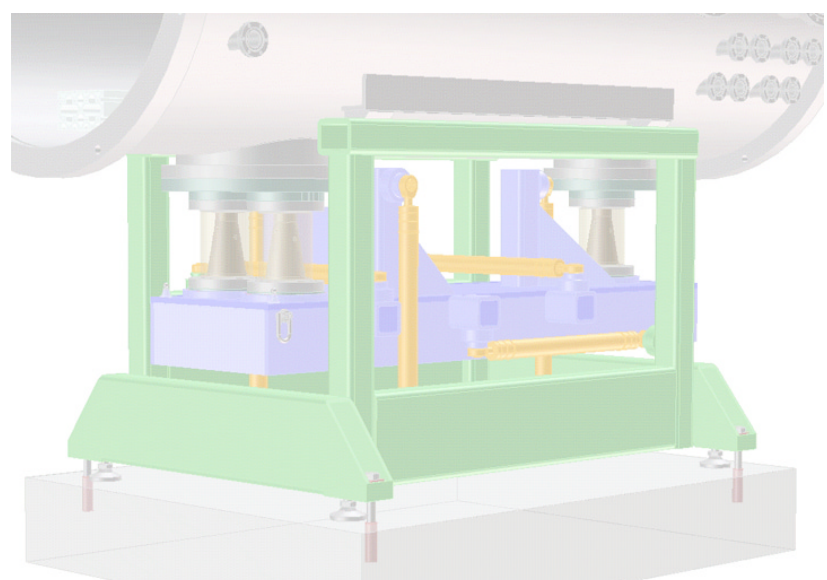

Figure 4: Vacuum vessel and support structure shown with the kinematic mounting system installed. The six strut system members are shown in yellow.

provides an alignment range of $\pm 25 \mathrm{~mm}$ with less than $12 \mu \mathrm{m}$ linear and $0.15 \mathrm{mrad}$ rotational resolution. Each of the bellows incorporates a bearing between the six-strut support and the vacuum feedthrough to allow alignment about the vertical axis without inducing torsion to the bellows (Fig. 5). 


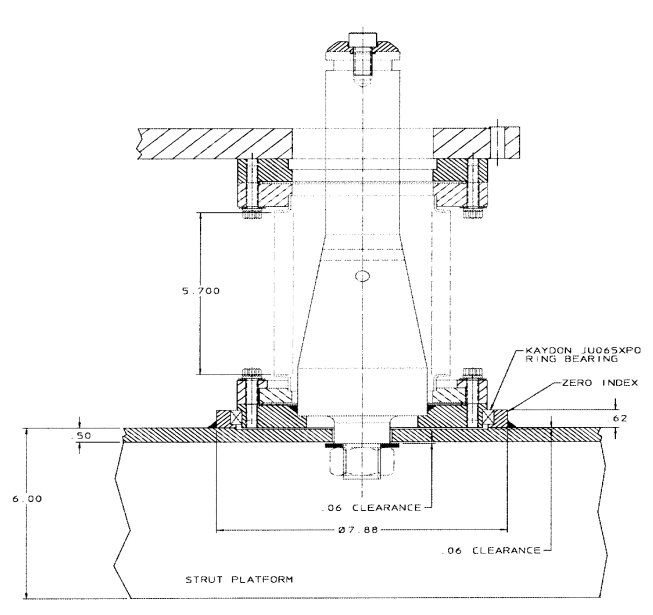

Figure 5: Detail of the bearing at the base of the support rod to prevent stressing the bellows during alignment.

\section{MECHANICALLY ADJUSTABLE ESQs}

Each ten ESQ assembly tank has two steering ESQs (at the last two lattice positions), and one combination diagnostic and rotatable ESQ (first lattice position). The steering ESQs are attached to the rail via vacuum compatible horizontal and vertical stage combinations that provide $\pm 12 \mathrm{~mm}$ translation with $12 \mu \mathrm{m}$ resolution. These translations allow steering of the beam centroid in both the horizontal and vertical planes since a displaced ESQ acts as a quadrupole of the same strength with a superimposed dipole (steering) field with strength proportional to the displacement. Movement is controlled externally through rotary feedthroughs. Displacing two adjacent ESQs allows full steering corrections in centroid and angle. The diagnostic ESQ translates horizontally out of the beam path to allow space for beam diagnostics mounted on the vacuum vessel. A horizontal stage and linear feedthrough provide the translation while under vacuum. Positive positioning features provide repeatable alignment. This ESQ can be rotated $\pm 4^{\circ}$ about the beam axis to provide variable skew coupling to the beam. Rotation is accomplished by accessing the ESQ manually through an access port, loosening bolts, turning the electrode plates, and re-securing the bolts.

\section{VACUUM VESSEL}

The cylindrical stainless steel vacuum vessel is $2286 \mathrm{~mm}$ long and $92 \mathrm{~mm}$ in diameter (Figs. 1 \& 4). There are 26 ports for diagnostics, high voltage feedthroughs, the kinematic support system, vacuum pumps, and maintenance access. The vessel will be pumped with an 8inch cryo pump providing a base pressure of less than $1 \times 10^{-7}$ Torr. Additional vacuum pumps can be accommodated, if necessary.

\section{DIAGNOSTICS}

Beam diagnostic stations are located at the first quadrupole of each tank. Each station has two-slit scanners for measurement of pulse resolved phase space projections of the beam distribution in the horizontal and vertical planes. These slit scanners can be used to measure the envelope of beam particles and the emittance of the beam along each plane. Attached to the downstream slit of each two-slit scanner is a Faraday cup to measure the transmitted beam current through the second slit. Each slit assembly is independently driven by a computer controlled step motor, with a positioning accuracy of $\sim 10 \mu \mathrm{m}$. The step motors reside outside the vacuum system, and drive the diagnostics via a ferrofluidic seal and lead screw assembly. The width of the slits in the scanning direction is $100 \mu \mathrm{m}$ or less, and each slit and slitFaraday cup pair is separated longitudinally by $10 \mathrm{~cm}$. Slit widths and drift distances are chosen such that the spacecharge expansion of the ribbon of beam that is transmitted through the first slit is $<10 \%$ of the expected expansion due to the local thermal velocity spread of the beam. Each diagnostic station also includes a large Faraday cup for accurate measurements of the total beam current. There are spare diagnostic ports for the implementation of new diagnostics currently under development. In addition to transverse beam profiles and phase space measurements in each of the transverse planes, measurements of the 2dimensional current density profile $\mathrm{J}(\mathrm{x}, \mathrm{y})$ will be inferred from measurements using "crossed slits" with one $\mathrm{x}$-slit with a y-slit. The beam parameters are stable from pulse to pulse allowing phase space to be measured with successive (20-200 shots at $\sim 0.1 \mathrm{~Hz}$ ) shots.

\section{STATUS}

The experimental hardware for the first tank of ESQs is scheduled for assembly, installation, and operation by October 2001. Contingent on funding, the subsequent 3 tanks will be fabricated and assembled in 2002 .

\section{ACKNOWLEDGEMENTS}

The authors wish to thank Peter Luft, Bob Manahan, and Vern Williamson for their contributions to the design of this apparatus and the mechanical and electrical technicians of the LBNL Heavy Ion Fusion program for their assembly and installation of the apparatus.

\section{REFERENCES}

[1] P. Seidl, et al, "Overview of the Scientific Objectives of the High Current Experiment for Heavy-Ion Fusion," these proceedings.

[2] F. Bieniosek, et al, "2-MV Injector for HCX," these proceedings.

[3] William Thur, "Rigid, Adjustable Support of Aligned Elements via Six Struts,” LBNL-40964, UC-406, 1997 